\title{
Prospective Assessment After Pediatric Cardiac Ablation: Design and Implementation of the Multicenter Study
}

\author{
GEORGE F. VAN HARE, * DORIT CARMELLI,† W. MCFATE SMITH,† \\ JOHN KUGLER,‡ MICHAEL SILKA,§ RICHARD FRIEDMAN,\| \\ DIANNE ATKINS,\# J. PHILIP SAUL, ${ }^{*}$ MICHAEL SCHAFFER,†† \\ CRAIG BYRUM,‡¥ ANN DUNNIGAN,§§ STEVEN COLAN,|\|| \\ GERALD SERWER,\#\# AND PARTICIPATING MEMBERS OF THE PEDIATRIC \\ ELECTROPHYSIOLOGY SOCIETY

\begin{abstract}
From the *Department of Pediatrics, Division of Cardiology, Stanford University, Stanford, +SRI International, Menlo Park, California, ¥University of Nebraska Medical Center, Omaha, Nebraska, §Children's Hospital of Los Angeles, University of Southern California, Los Angeles, California, ||Texas Children's Hospital, Baylor College of Medicine, Houston, Texas, \# University of Iowa, Iowa City, Iowa, ** Medical University of South Carolina, Charleston, South Carolina, +†Denver Children’s Hospital, Denver, Colorado, ¥¥State University of New York-Syracuse Health Science Center, Syracuse, New York, §§Minneapolis Heart Institute, Minneapolis, Minnesota, ||||Children’s Hospital, Boston, Massachusetts, and \#\#University of Michigan-CS Mott Children's Hospital, Ann Arbor, Michigan
\end{abstract}

\begin{abstract}
VAN HARE, G.F., ET Al.: Prospective Assessment After Pediatric Cardiac Ablation: Design and Implementation of the Multicenter Study. A multicenter prospective study was designed and implemented as an activity of the Pediatric Electrophysiology Society to assess the risks associated with radiofrequency ablation in children. Patients (age 0-15 years) with supraventricular tachycardia due to accessory pathways or atrioventricular nodal reentry were enrolled and studied prior to ablation and periodically by clinical evaluation, electrocardiogram (ECG), Holter monitor, and echocardiogram. In addition, a national registry was established, to which the contributing centers report all pediatric patients undergoing ablation at their center. Initial electrophysiological study tracings and all noninvasive studies undergo blinded outside review for quality control. Clinical endpoints were death, recurrence, proarrhythmia, and echocardiographic abnormality. A pilot study demonstrated excellent agreement concerning diagnoses of previously reported ablation patients between the reporting center and the blinded reviewer (kappa $=0.938 \pm$ 0.062). A total of 317 patients were enrolled in the ongoing study from April 1, 1999 to December 31, 2000. The success rate of ablations was $96 \%$ with a complication rate of $4.3 \%$ for electrophysiological study and $2.9 \%$ for the ablation procedure. Comparison of the registry group versus the study group shows that the groups are comparable in terms of patient characteristics, diagnoses, and the results of ablation making it less likely that the sample of prospectively enrolled patients is biased. (PACE 2002; 25:332-341)
\end{abstract}

ablation, children, Wolff-Parkinson-White syndrome, atrioventricular nodal reentry, registry

\section{Introduction}

Radiofrequency catheter ablation is a technique that can be used to eliminate cardiac arrhythmias, and it has become common in pediatric cardiology practice. Recent analyses have

Supported in part by R01 HL58620 from the National Heart, Lung, and Blood Institute, National Institutes of Health.

Address for reprints: George F. Van Hare, M.D., Pediatric Cardiology, Stanford University, 750 Welch Rd., Rm 305, Palo Alto, CA 94304. Fax: (650) 725-8343; e-mail: vanhare@Stanford.edu

Received March 27, 2001; accepted April 5, 2001. suggested that ablation therapy of children with Wolff-Parkinson-White syndrome (WPW) is more cost effective when compared with surgery or antiarrhythmic drug therapy. ${ }^{1}$ Reported success rates for the technique have been high and complication rates low ${ }^{2}$; however, there remains concern about possible long-term problems with the technique in the pediatric age group. Cardiac valve damage, the development of new arrhythmias, and late sudden death have been reported in children. ${ }^{2,3}$ Recurrences are observed frequently following initially successful procedures. Finally, there is animal data to suggest that immature myocardium may be more prone to severe damage as a result of ablation procedures. ${ }^{4}$ Few if any data 
exist to support the long-term safety of these ablation techniques in children. Therefore, it is critically important to carefully assess the long-term risks in this patient population.

To better assess these risks and the potential for recurrence, a multicenter prospective study was designed and implemented as an activity of the Pediatric Electrophysiology Society. This project was conceived as an extension of the Pediatric Radiofrequency Ablation Registry (PRAR), a voluntary registry of pediatric patients undergoing catheter ablation which was established in 1991. The PRAR has previously reported results from procedures in children, ${ }^{2,5-7}$ but has not included any prospective data or included the results of noninvasive evaluations. This report describes the design, implementation, and initial findings of the Prospective Assessment after Pediatric Cardiac Ablation (PAPCA) study. Further data will be published in the future as the data set is completed and data analysis is carried out.

\section{Methods}

A multicenter prospective study was initiated with patients enrolled by the contributing centers of the Pediatric Electrophysiology Society. A total of 41 separate centers have agreed to participate in PAPCA to date (Appendix I). Patients who meet the predefined inclusion criteria (below) for enrollment are identified at each of the contributing centers, which have each obtained approval of the protocol from their local Institutional Review Board. This group is referred to as "PAPCA patients." In addition, to allow for a comparison of the study population with the other populations with respect to sex, race, diagnosis, age, and outcome, a national registry was established, to which the contributing centers report all pediatric patients undergoing ablation at their center.

\section{Specific Aims and Hypotheses}

The specific aims of the study tried to answer, by the use of a prospective design, the following three questions in the pediatric population (Table I):

Table I.

Specific Aims of the Prospective Assessment After Pediatric Cardiac Ablation Study

To determine in the pediatric population:

1. Incidence and time course of recurrence of an initially successfully ablated arrhythmia

2. Incidence of early and late-appearing cardiac damage due to the ablation procedure

3. Incidence of new arrhythmias attributable to the ablation procedure
1. What is the incidence and time course of recurrence following the initially successful ablation of an arrhythmia? Specifically, the hypotheses to be tested were that the rate of recurrence as assessed by clinical information and ECGs at 2year postablation $<20 \%$, and that there is no difference in rate of recurrence at 2-year postablation between patients with accessory pathways in the right free wall versus the left free wall.

2. What is the incidence of early and late appearing cardiac damage due to the ablation procedure? Specifically, the hypotheses to be tested were that the incidence of damage to cardiac valves and other structures detected at 2-month postablation is $<5 \%$, and that the incidence of late appearing cardiac damage, like cardiac functional abnormalities and/or aneurysm formation, assessed echocardiographically at 2-year postablation, is $<1 \%$.

3. What is the incidence of new arrhythmias attributable to the ablation procedure? Specifically, the hypothesis to be tested was that the incidence of late appearing new arrhythmias, detected by 24-hour Holter monitors at 2-year postablation, is $<5 \%$.

\section{Clinical Endpoints}

The following clinical endpoints were defined as study outcomes.

1. Death: As determined by initial or followup data forms, or search of the National Death Index. Each death was classified as "related to the ablation," "not related to the ablation," or of "uncertain relation to ablation." This determination was made by the Morbidity/Mortality Review Committee.

2. Recurrence: As determined by each individual investigator at the time of follow-up, and based on the presence or absence of preexcitation for WPW patients and/or documented recurrence of symptomatic tachycardia for patients with or without WPW.

3. Proarrhythmia: As determined by the comparison of the results from 24-hour Holter monitors obtained prior to the ablation and at intervals following the ablation. Holters are scored with an ordinal scale ranging from 1 (normal) to 5 (most abnormal). Proarrhythmia is defined by the appearance of an abnormality following ablation that was not present prior to ablation, for example, the appearance of premature ventricular contractions following ablation. The Morbidity/Mortality Review Committee makes the final judgment on whether the findings represent "proarrhythmia definite," "proarrhythmia possible," or "proarrhythmia absent."

4. Echocardiographic Abnormality: As determined by comparisons of readings for each 


\section{Table II.}

Inclusion/Exclusion Criteria for the Prospective Study

Inclusion criteria:

1. Children undergoing ablation age $0-16$ at the time of the ablation

2. Symptomatic paroxysmal tachycardia due to an accessory pathway (Wolff-Parkinson-White syndrome, concealed accessory pathway, or persistent junctional reciprocating tachycardia) or to atrioventricular node reentrant tachycardia

3. Otherwise normal heart

Exclusion criterion

1. Significant congenital heart disease, other than a small (hemodynamically insignificant) atrial septal defect, small (hemodynamically insignificant) ventricular septal defect, small (hemodynamically insignificant) patent ductus arteriosus or patent foramen ovale)

echocardiographic parameter, and by using an ordinal scale ranging from 1 (normal, without valve regurgitation or wall-motion abnormality) to 4 (most abnormal).

\section{Inclusion/Exclusion Criteria}

Children undergoing ablation who were age 0-16 years at the time of the ablation were eligible for enrollment (Table II). Patients with symptomatic paroxysmal supraventricular tachycardia due to an accessory pathway (WPW syndrome, concealed accessory pathway, the persistent form of junctional reciprocating tachycardia), or to atrioventricular nodal reentrant tachycardia (AVNRT) were included. Patients were excluded if they had additional arrhythmia mechanisms other than the ones stated above, and if there was significant congenital heart disease, other than a small atrial septal defect, ventricular septal defect, or patent foramen ovale.

\section{Clinical Procedures}

After the patients' families give informed consent, and prior to the ablation procedure, patients underwent noninvasive testing, consisting of an ECG, 24-hour ambulatory ECG (Holter monitor), and two-dimensional echocardiogram with color Doppler interrogation. For each procedure performed, the cardiologist at the contributing center fills out an initial data form that includes: patient demographics (with race and sex), indication for ablation, diagnosis and pathway location, method of ablation, number of applications of radiofrequency energy, fluoroscopy exposure and procedure times, and initial success and complication data. At intervals following the ablation procedure, subjects return to the contributing centers for clinical follow-up, at which time additional clinical details are collected and reported (symptoms, medications, physical examination findings, complications) and additional ECGs, Holter monitors, and echocardiograms are obtained. The final follow-up visit occurs 2 years after ablation. The full schedule is found in Table III. For all noninvasive studies, local investigators interpret the test and record the interpretation on standard forms.

\section{Data Coordinating Center}

Data collection, quality control, and analysis are performed by the Data Coordinating Center, based at the Center for Health Sciences of SRI International in Menlo Park, California.

\section{Quality Control}

Central reading centers were established for echocardiograms, ECGs, electrophysiological study tracings, and Holter monitors. Expert reviewers interpret the results of the noninvasive tests to ensure standardized interpretations of high quality. In addition, an electrophysiology reviewer reviews a randomly selected subset of consisting of $50 \%$ of initial electrophysiological studies of enrolled patients, to ensure that the

Table III.

Schedule of Clinical Procedures

\begin{tabular}{lccccc}
\hline Test/Interval & Enrollment & $\begin{array}{c}\mathbf{2} \\
\text { Months }\end{array}$ & $\begin{array}{c}\mathbf{6} \\
\text { Months }\end{array}$ & $\begin{array}{c}12 \\
\text { Months }\end{array}$ & $\begin{array}{c}\mathbf{2 4} \\
\text { Months }\end{array}$ \\
\hline Initial data form & $\mathrm{X}$ & & & & \\
Follow-up form & $\mathrm{X}$ & $\mathrm{X}$ & $\mathrm{X}$ & $\mathrm{X}$ & $\mathrm{X}$ \\
ECG & $\mathrm{X}$ & $\mathrm{X}$ & $\mathrm{X}$ & $\mathrm{X}$ & $\mathrm{X}$ \\
Holter & $\mathrm{X}$ & $\mathrm{X}$ & & & $\mathrm{X}$ \\
Echo & & & & $\mathrm{X}$ \\
\hline
\end{tabular}

$\mathrm{ECG}=$ electrocardiography. 
enrollment criteria for patients have been met. The reviewers are blinded with respect to the diagnosis, ablation site, ablation result, and whether the study is pre- or postablation. Error-free data entry into an analysis database is assured by the Data Coordinating Center by using double entry techniques with error checking.

\section{Diagnostic Criteria}

The electrophysiological diagnosis is confirmed by each investigator at the time of the ablation and must meet standard criteria for the diagnosis (Appendix II). These diagnostic criteria are also used by the electrophysiological consultant for quality control reviews.

Because each center only recruits voluntary patients undergoing ablation at that site, it is important to assess the potential bias in this selection procedure. The registry is used to monitor the demographic and diagnostic data on all patients undergoing ablation at each participating center, including those enrolled in PAPCA and those who are not enrolled.

\section{Sample Size Determinations}

Sample size calculations were performed for the primary outcome variables in PAPCA: the recurrence rate postablation, early versus late incidence rates of cardiac damage, and the incidence of new arrhythmias. ${ }^{8}$ Previous studies have reported recurrence risks from $15 \%$ to $50 \% .^{5,9,10}$ The authors expect the rate of recurrence in the present study to be $<20 \%$. They have estimated that with a sample of $n=450$, they can obtain $95 \%$ confidence intervals that range from $16.3 \%$ to $23.7 \%$ if the recurrence rate is $20 \%$.

For a comparison of the recurrence rates of subjects with left versus right free-wall accessory pathway, 264 of 450 patients were assumed to have left or right free-wall pathways as opposed to AVNRT or other pathway locations, and that a distribution of $25 \%$ and $75 \%$ right- versus left-sided locations will hold, based on the latest proportions reported by the PRAR. This sample size gave the study $80 \%$ power to detect an absolute difference in recurrence of $16 \%$ with $95 \%$ confidence.

In the preliminary study by the principal investigator,$^{10}$ the incidence of late abnormalities on Holter was found to be $5 \%$. Considering an increase of $10 \%$ in the rate of late arrhythmia as clinically important, the sample size needed to detect such an increase or larger is $\mathrm{n}=404$ to assure $90 \%$ power with $95 \%$ accuracy.

\section{Simultaneous Registry}

A simultaneous registry was established for the purpose of ascertaining the pediatric population undergoing ablation. This registry was de- signed to replace and extend to all the functions of the previous PRAR. Participating centers report data from all ablation procedures in patients $<21$ years of age with any electrophysiological mechanism with or without congenital heart disease. Therefore, the inclusion criteria are much broader for the registry than for PAPCA (Table IV). In addition, while all PAPCA patients were required to give informed consent prior to participation, two routes for participation were established for the registry patients. Patients can be enrolled after giving informed consent, in which case patient identifying data is provided to the Data Coordinating Center. Alternatively, procedures can be reported without any patient identifying data, eliminating the need for obtaining informed consent. The initial data form is filled out for each patient, and limited follow-up data is submitted for these patients, without the requirement for noninvasive studies.

\section{Pilot Study}

A pilot project was performed in July and $\mathrm{Au}-$ gust 1996 by the principal investigator, the Data Coordinating Center, and one of the expert consultants (AD). The purpose was to test the quality of the data previously submitted to the PRAR and to test the procedures proposed for quality control. This pilot project assessed, by independent review, if the electrophysiological diagnoses of patients reported to the registry were correct. Six centers were recruited, of which three were relatively large and three were relatively small (Appendix III), and a list of all ablations reported to the registry from 1993-1996 was obtained from the registry. These six lists were randomized, and the first ten procedures were selected from each center. Electrophysiological tracings were coded and assembled in random order and forwarded to the consultant, who interpreted each study as to the preablation diagnosis. A study in some cases demonstrated more than one diagnosis. Because patients with AVNRT and with accessory pathways were included in PAPCA and other diag-

Table IV.

Inclusion/Exclusion Criteria for the Registry

Inclusion criteria:

1. Children undergoing radio frequency ablation age $0-21$ at the time of ablation

2. Symptomatic tachycardia due to any mechanism

3. Normal heart or with associated congenital heart disease

Exclusion criterion

1. Age $>21$ years 
Table V.

Gender and Minority Status of Pediatric Ablation Population

\begin{tabular}{|c|c|c|c|}
\hline Characteristic & $\begin{array}{c}\text { Pediatric Registry } \\
(1991-97, \mathrm{n}=1586)\end{array}$ & $\begin{array}{l}\text { PAPCA Enrollment } \\
(1999-2000, n=317)\end{array}$ & $\begin{array}{l}1990 \text { US } \\
\text { Census }\end{array}$ \\
\hline Male & $876(55 \%)$ & $166(52 \%)$ & $51 \%$ \\
\hline Female & $710(45 \%)$ & $151(48 \%)$ & $49 \%$ \\
\hline White, not of Hispanic origin & $1303(82 \%)$ & $269(85 \%)$ & $75 \%$ \\
\hline Black, not of Hispanic origin & $108(7 \%)$ & $26(8 \%)$ & $15 \%$ \\
\hline Hispanic, others & $147(9 \%)$ & $15(5 \%)$ & $6 \%$ \\
\hline Asian, Pacific Islander & $13(1 \%)$ & $5(2 \%)$ & $3 \%$ \\
\hline Native American & $15(1 \%)$ & $1(0.3 \%)$ & $1 \%$ \\
\hline
\end{tabular}

PAPCA $=$ Prospective Assessment After Pediatric Cardiac Ablation Study.

noses were to be excluded, the most important question was the reliability of the include or exclude decision. The final interpretations were compared by the Data Coordinating Center with a list of diagnoses reported to the registry.

\section{Sex and Minority Enrollment}

The project includes both sex and minority subjects. To ensure that female and minority subjects were included in the study, the authors surveyed participating centers of the PRAR for the sex and ethnic composition of the groups of patients reported to the PRAR (Table V). The results were compared with the data available from the 1990 United States Census for ages 0-21 years. Adequate numbers of patients from each sex and racial/ethnic origin category were available for inclusion in this study, and recruitment goals were established so that the distribution of patients selected would closely match that of the 1990 US Census figures for the age group to be studied, 0-15 years.

\section{Data Analysis}

All data analyses were conducted by the Data Coordinating Center at the Center for Health Sciences at SRI International. Analyses were carried out using the SAS (Cary, NC, USA) statistical software.

\section{Approval and Oversight}

The protocol received approval from the Institutional Review Boards of Stanford University and of all of the Contributing Centers. Additional oversight was provided by a Data and Safety Monitoring Committee, initiated by the principal investigator and composed of five independent advisors, which meets semiannually to review the progress of the trial, preliminary results, and complications.

\section{Finances}

This trial is funded by an individual research grant from the National Heart, Lung and Blood Institute, R01-HL58620. The Data Coordinating Center was funded through a subcontract that was formed between Stanford University and SRI International. Contributing Centers were reimbursed for the provision of data on prospective study patients, but not Registry patients, according to the schedule (Table III). Funding covered the provision of completed data forms and some monies for the Holter, ECG, and echocardiogram studies. Consultants were reimbursed on a per study basis.

\section{Results}

Preliminary results from the project are reported here, and comprise the results of the multicenter pilot study, early data from the trial, and initial quality control results.

\section{Pilot Study}

Table VI shows the results of the comparison between the blinded reviewer and the centers with respect to the decision whether to include or exclude a patient based on the diagnosis. Kappa is a measure for comparing the agreement of ob-

Table VI.

Pilot Study: Comparison of Include/Exclude Decisions

\begin{tabular}{lccc}
\hline & \multicolumn{2}{c}{ Reviewer } & \\
\cline { 2 - 3 } Original Site & Include & Exclude & Total \\
\hline Include & 50 & 1 & 51 \\
Exclude & 0 & 9 & 9 \\
Total & 50 & 10 & 60 \\
\hline
\end{tabular}


Table VII.

Pilot Study: Comparison of Diagnostic Group Comparisons

\begin{tabular}{lccccc}
\hline & \multicolumn{5}{c}{ Reviewer } \\
\cline { 2 - 6 } Original Site & AVNRT & AVRT & Other & $\begin{array}{c}\text { AVNRT } \\
\text { Vs AVRT }^{*}\end{array}$ & Total \\
\hline AVNRT (include) & 11 & 0 & 1 & 0 & 12 \\
AVRT (include) & 1 & 37 & 0 & 1 & 39 \\
Other (exclude) & 12 & 0 & 9 & 0 & 9 \\
Total & 37 & 10 & 1 & 60 \\
\hline
\end{tabular}

AVNRT = atrioventricular nodal reentrant tachycardia; AVRT $=$ atrioventricular reentrant tachycardia.

servers after taking into account the effect of chance alone. ${ }^{11}$ Zero represents no agreement beyond chance, 1 represents complete agreement. The kappa value for agreement on Table VI is 0.938 , with a standard error of 0.062 . According to the characterization of Landis and Koch, ${ }^{12}$ a kappa of this magnitude may be taken to represent excellent agreement between the observers.

The comparison in terms of diagnostic groups is shown in Table VII. The reviewer felt that in one case, the electrophysiological data provided was insufficient to distinguish between AVNRT and atrioventricular reentrant tachycardia (AVRT), so this case was treated as a disagreement, the most conservative option. The overall kappa for this Table VII is 0.906 with a standard error of 0.052 , indicating excellent agreement. Kappa scores for each category individually are consistent with the overall kappa (0.896 for AVNRT, 0.955 for AVRT, and 0.938 for Other).

\section{Sex/Minority Enrollment in Previous PRAR}

Twenty-one United States centers provided data on 1,586 patients previously enrolled into the registry, which represents $74 \%$ of patients reported to the registry for the requested years. Patients of all races and sexes have been reported to the registry, with some under-representation of Asians and Blacks, and some over-representation of Whites and Hispanics, when compared with 1990 US Census data (Table V).

\section{Early Results}

\section{Patient Population}

Recruitment into PAPCA began April 1, 1999. Early results represent data accumulated during the first 21 months of operation until December 31,2000 . A total of 317 patients were enrolled (Tables V, VIII) of which $52 \%$ were male, and $15 \%$ were nonwhite and/or Hispanic. Based on 1990 US Census data, in which males make up 51\% of the 0-15-year-old population, females are not significantly under-represented in this study. However, nonwhites are under-represented, making up $25 \%$ of the same population.

\section{Results of the Ablation Procedures}

Overall, investigators at the contributing centers have achieved a $96 \%$ success rate initially for ablation of supraventricular tachycardia (SVT) in the prospective study. The success rates by substrate are listed in Table IX. Complications of the electrophysiological studies occurred in $4.3 \%$ of procedures, while complications of radiofrequency ablation occurred in $2.9 \%$ of the procedures (Table X). These results are similar to those reported by the PRAR. ${ }^{2}$

\section{Table VIII.}

Patient Demographics

\begin{tabular}{lcc}
\hline & PAPCA & Registry \\
\hline Mean age years & $11.3 \pm 3.7$ & $13.1 \pm 4.2$ \\
Mean weight kg & $44.6 \pm 17.9$ & $52.2 \pm 20.2$ \\
Sex \% of male & $52 \%$ & $56 \%$ \\
$\begin{array}{l}\text { Structural heart disease } \\
\quad \text { Congenital }\end{array}$ & $2 \%$ & $11 \%$ \\
$\quad$ Cardiomyopathy & $1 \%$ & $2 \%$ \\
Indication for ablation & & \\
$\quad$ Life-threatening & $7 \%$ & $10 \%$ \\
$\quad$ arrhythmia & & \\
$\quad$ Refractory to drug & $15 \%$ & $16 \%$ \\
$\quad$ treatment & & \\
$\quad$ Adverse drug effects & $1 \%$ & $2 \%$ \\
$\quad$ Impending CHD surgery & 0 & $2 \%$ \\
$\quad$ Patient choice & $75 \%$ & $68 \%$ \\
$\quad$ Cardiomyopathy & $1 \%$ & $2 \%$ \\
Fluoroscopy Time & $39.9 \pm 31.0$ & $36.7 \pm 34.2$ \\
\hline
\end{tabular}

PAPCA $=$ Prospective Assessment After Pediatric Cardiac Ablation Study; $\mathrm{CHD}=$ congenital heart disease. 
Table IX.

Results of Ablation

\begin{tabular}{lcc}
\hline \multicolumn{1}{c}{ Diagnoses } & PAPCA & Registry \\
\hline Accessory pathways & $193 / 202^{*}(95 \%)$ & $467 / 493(95 \%)$ \\
AVNRT & $67 / 68(98 \%)$ & $184 / 194(95 \%)$ \\
Atrial fibrillation & - & $1 / 1(100 \%)$ \\
AET & - & $41 / 42(98 \%)$ \\
Atrial flutter & - & $30 / 34(88 \%)$ \\
JET & - & $42 / 42(100 \%)$ \\
VT & - & $17 / 22(77 \%)$ \\
Other & $260 / 270(96 \%)$ & $787 / 836(92 \%)$ \\
Total &
\end{tabular}

* Success rates computed on a smaller cohort due to missing data in mid-trial. See previous tables for abbreviations. AET $=$ atrial ectopic tachycardia; JET = junctional ectopic tachycardia.

\section{Registry Versus Prospectively Enrolled Patients}

When patients enrolled in PAPCA were compared with those entered into the registry, several differences in demographic characteristics were noted. Most of these are due to different inclusion/exclusion criteria. Specifically, the registry includes patients up to 21 years of age and includes all substrates and patients with congenital heart disease. However, when the registry patients who would otherwise be eligible for enrollment in the prospective study (based on age, substrate, and structurally normal heart) were analyzed separately, most of these differences disappeared (Table X).

\section{Discussion}

The current study offers significant potential advantages over prior published registry-style studies. This study is, in some respects, similar to the successful Percutaneous Cardiac Mapping and Ablation Registry (PCMAR), which studied the effect of direct current catheter ablation in the adult population. ${ }^{13}$ That project identified sudden death as a late consequence of direct current ablation in $1.6 \%$ of patients, a somewhat unexpected finding. Calkins et al. ${ }^{14}$ reported the results of a prospective trial involving the Medtronic Atakr Ablation System (Minneapolis, MN, USA), and reported on 1,050 patients who underwent radiofrequency ablation. While having the advantage of being a careful prospective trial, outside review of diagnoses, echocardiograms, and other materials were not performed. Furthermore, despite the plan for follow-up out to 24 months following ablation, the mean follow-up was only 6.3 months. Finally, Scheinman and Huang ${ }^{15}$ recently reported initial data from the North American Society of Pacing and Electrophysiology (NASPE) Prospective Catheter Ablation Registry. Unlike the current project, the NASPE Registry is entirely voluntary and there is no funding for follow-up studies, no outside review of findings, and no plan for quality control. Furthermore, only 68 centers are participating out of a known national group of 950 centers currently performing ablation procedures. The specific potential advantages of the current study include nearly full national ascertainment of pediatric procedures, rigorous quality control procedures for data entry and diagnosis of

Table X.

PAPCA versus PAPCA-eligible Registry Patients

\begin{tabular}{|c|c|c|c|}
\hline & PAPCA & $\begin{array}{l}\text { PAPCA-Eligible } \\
\text { Registry }\end{array}$ & $\begin{array}{c}\text { PAPCA-Noneligible } \\
\text { Registry }\end{array}$ \\
\hline Age, yr & 11.34 & 12.20 & 15.79 \\
\hline Weight, kg & 44.57 & 48.81 & 61.94 \\
\hline Substrate: WPW & $117(43.8 \%)$ & $213(40.6 \%)$ & 56 (35.7\%) \\
\hline Concealed AP & $73(27.3 \%)$ & $158(30.1 \%)$ & $43(27.4 \%)$ \\
\hline PJRT & $9(3.4 \%)$ & $14(2.7 \%)$ & $1(0.64 \%)$ \\
\hline AVNRT & $68(25.5 \%)$ & $139(26.5 \%)$ & 57 (36.3\%) \\
\hline \multicolumn{4}{|l|}{ Pathway location: } \\
\hline Right-sided (sites 1-6) & $70(34.8 \%)$ & $150(40.5 \%)$ & 48 (49.5\%) \\
\hline Left-sided (sites 7-11) & $131(65.2 \%)$ & $220(59.5 \%)$ & 49 (50.5\%) \\
\hline Results: \#successful & 259/269 (96\%). & $498 / 525(95 \%)$ & $150 / 156(96 \%)$ \\
\hline $\begin{array}{l}\text { \# with electrophysiological } \\
\text { study complications }\end{array}$ & $12 / 277(4.3 \%)$ & $21 / 540(3.9 \%)$ & $8 / 161(5.0 \%)$ \\
\hline \# with ablation complications & $8 / 277$ (2.9\%) & $14 / 54(2.6 \%)$ & $6 / 161(3.7 \%)$ \\
\hline
\end{tabular}




\section{PROSPECTIVE ASSESSMENT AFTER PEDIATRIC CARDIAC ABLATION}

complications, the performance of routine noninvasive studies in all enrolled patients, and the prospect of more complete follow-up than has been reported previously.

\section{Pilot Study}

When proposing or initiating a multicenter clinical trial, it is important to demonstrate the ability of the individual centers to successfully enroll patients. The importance of this aspect of study design was demonstrated in the results of the original PRAR. ${ }^{5}$ However, the accuracy of the major inclusion criterion (electrophysiological diagnosis) was never independently assessed in that study. In contrast, the results of the current pilot study provided evidence that (1) patients entered in the PRAR were by and large diagnosed correctly prior to ablation, and (2) the frequency of disagreement between the registry diagnoses and the electrophysiology consultant was likely to be sufficiently small as to not affect data and subgroup analyses. Finally, this pilot project demonstrated the ability of the principal investigator, the data coordinating center, and the current registry members to conduct the type of detailed quality control procedures that are needed in the study.

\section{Sex/Minority Enrollment}

Multiple factors may account for the fact that the distribution does not exactly match the 1990 US Census figures, like differing access to tertiary medical care services and geographic variation in racial/ethnic origin distribution. However, enrollment by race and sex was similar to the pattern observed during the original PRAR (Table VIII) suggesting that there is no inherent statistical bias introduced by the process of obtaining consent and entering patients into the current study. The current study is, in fact, a good representation, at least by race and sex, of the larger population that has previously undergone ablation and was reported to the original registry. It has long been known that SVT is more common in male than female infants. ${ }^{16,17}$ However, careful studies of the actual incidence of SVT in children subdivided by race have not been done, and so it is possible that the under-representation of minority subjects results from differing ethnic incidence of the disease. Interestingly, a recent large study of adults with atrial fibrillation found relative under-representation of nonwhites. ${ }^{18}$ Short of large epidemio- logical prevalence studies, these series may represent the only estimates of the incidence of these diseases by race and sex in the pediatric population. Despite these considerations, it is the intention of this study to actively continue to attempt to recruit female subjects, and subjects from underrepresented ethnic groups into the prospective study.

\section{Early Results}

The early findings, comprising success rates and complication rates, are similar to those previously reported. This finding is reassuring, as it suggests that the cohort of patients enrolled in this study will be representative of the pediatric population undergoing ablation in the United States. It is too early to report the results of patient followup including the results of noninvasive testing. As this information becomes available, we will be able to estimate the actual frequency and time course of recurrence after an initially successful ablation, and to assess the long-term risks of the procedure.

\section{Overall Significance of the Project}

The state of scientific knowledge considering clinical practice is quite different when one compares the practice of adult electrophysiology to the practice of the same discipline in children. In adults, much of current clinical practice has been evaluated by means of prospective trials, like the Amiodarone versus Implantable Defibrillator (AVID) trial ${ }^{19}$ and others. In children, however, multicenter research tends to be retrospective. Concerns about patient size, developmental differences between adults and children, and different comorbidities persuade one that studies performed in adults are not necessarily applicable to the pediatric population. This is the first funded multicenter prospective clinical study of children with arrhythmias. As such, it has established a network of centers that are experienced in the performance of clinical research. The experience gained will allow the network to address other issues in pediatric arrhythmia management.

Acknowledgments: The authors thank Gaye Courtney, Clinical Study Coordinator, and Ruth Krasnow, Data Manager, both of SRI International, for their diligent work in the launching of this clinical study.

\section{References}

1. Garson A, Kanter RJ. Management of the child with Wolff-Parkinson-White syndrome and supraventricular tachycardia: Model for cost effectiveness. J Cardiovasc Electrophysiol 1997; 8:1320-1326.

2. Kugler JD, Danford DA, Houston K, et al. Radiofrequency catheter ablation for paroxysmal supraventricular tachycardia in children and adolescents without structural heart disease. Pediatric EP So-

ciety, Radiofrequency Catheter Ablation Registry. Am J Cardiol 1997; 80:1438-1443.

3. Schaffer MS, Gow RM, Moak JP, et al. Mortality following radiofrequency catheter ablation (from the Pediatric Radiofrequency Ablation Registry). Participating members of the Pediatric Electrophysiology Society. Am J Cardiol 2000; 86:639-643. 
4. Saul JP, Hulse JE, Papagiannis J, et al. Late enlargement of radiofrequency lesions in infant lambs. Implications for ablation procedures in small children. Circulation 1994; 90:492-499.

5. Kugler JD, Danford DA, Deal BJ, et al. Radiofrequency catheter ablation for tachyarrhythmias in children and adolescents. N Engl J Med 1994; 330:1481-1487.

6. Danford DA, Kugler JD, Deal B, et al. The learning curve for radiofrequency ablation of tachyarrhythmias in pediatric patients. Participating members of the Pediatric Electrophysiology Society. Am J Cardiol 1995; 75:587-590.

7. Schaffer MS, Silka MJ, Ross BA, et al. Inadvertent atrioventricular block during radiofrequency catheter ablation. Results of the Pediatric Radiofrequency Ablation Registry. Pediatric Electrophysiology Society. Circulation 1996; 94:3214-3220.

8. Kraemer H, Thiemann S. How Many Subjects? Statistical Power Analysis in Research. Beverly Hills, Sage Publications, 1987.

9. Minich LL, Snider AR, Dick Md. Doppler detection of valvular regurgitation after radiofrequency ablation of accessory connections. Am J Cardiol 1992; 70:116-1167.

10. Van Hare GF, Witherell CL, Lesh MD. Follow-up of radiofrequency catheter ablation in children: Results in 100 consecutive patients. J Am Coll Cardiol 1994; 23:1651-1659.

11. Fleiss JL. Statistical Methods for Rates and Proportions. 2nd ed. New York, John Wiley \& Sons, 1981.

\section{Appendix I}

\section{Participants and Contributing Centers}

Macdonald Dick, II, M.D., University of Michigan-CS Mott Children's Hospital, Ann Arbor, MI; Robert Campbell, M.D., Eggleston Children's Hospital, Atlanta, GA; Yung R. Lau, M.D., University of Alabama, Birmingham, AL; Edward P. Walsh, M.D., Children's Hospital, Boston, MA; J. Philip Saul, M.D., Medical University of South Carolina, Charleston, SC; Timothy Knilans, M.D., Children's Hospital Medical Center, Cincinnati, $\mathrm{OH}$; William Scott, M.D., Children's Medical Center, Dallas, TX; Jeanny Park, M.D., University of California Davis, Davis, CA; Michael S. Schaffer, M.D., Children's Hospital, Denver, Denver, CO; Peter Karpawich, M.D., Children's Hospital of Michigan, Detroit, MI; Ronald Kanter, M.D., Duke University, Durham, NC;

Margaret Bell, M.D., Arrhythmia Associates, Fairfax, VA; Richard Friedman, M.D., Texas Children's Hospital, Houston, TX; Steven Weindling, M.D., Dartmouth-Hitchcock Medical Center, Lebanon, NH;

Christopher Erickson, M.D., Arkansas Children's Hospital, Little Rock, AR; Ruchir Sehra, M.D., Loma Linda University Medical Center, Loma Linda, CA; Kevin M. Shannon, M.D., UCLA Children's Hospital, Los Angeles, CA; Ming-Lon Young, M.D., University of Miami, Miami, FL; Ann Dunnigan, M.D., Minneapolis Heart Institute, Minneapolis, MN; Frank Fish, M.D., Vanderbilt University, Nashville, TN; Steven Fishberger, M.D., Children's Hospital of New York, New York, NY;

Bertrand Ross, M.D., Children's Hospital of the King's Daughters, Norfolk, VA; John Kugler, M.D., University of Nebraska, Omaha, NE;
12. Landis JR, Koch GG. The measurement of observer agreement for categorical data. Biometrics 1977; 33:159-174.

13. Evans GT, Scheinman MM, Zipes DP, et al. The Percutaneous Cardiac Mapping and Ablation Registry: Final summary of results. PACE 1988; 11:1621-1626.

14. Calkins H, Yong P, Miller JM, et al. Catheter ablation of accessory pathways, atrioventricular nodal reentrant tachycardia, and the atrioventricular junction: Final results of a prospective, multicenter clinical trial. The Atakr Multicenter Investigators Group. Circulation 1999; 99:262-270.

15. Scheinman MM, Huang S. The 1998 NASPE prospective catheter ablation registry. PACE 2000; 23:1020-1028.

16. Garson A, Gillette PC, McNamara DG. Supraventricular tachycardia in children: Clinical features, response to treatment, and long-term follow-up in 217 patients. J Pediatr 1981; 98: 875-882.

17. Lundberg A. Paroxysmal atrial tachycardia in infancy: Long-term follow-up study of 49 subjects. Pediatrics 1982; 70:638-642.

18. Gibbs CR, Lip GY. Atrial fibrillation and ethnicity. Circulation 1999; 100:e153.

19. A comparison of antiarrhythmic-drug therapy with implantable defibrillators in patients resuscitated from near-fatal ventricular arrhythmias. The Antiarrhythmics versus Implantable Defibrillators (AVID) Investigators. N Engl J Med 1997; 337:1576-1583.

Anne M. Dubin, M.D., Lucile Packard Children's Hospital at Stanford, Palo Alto, CA; Ronn Tanel, M.D., Children's Hospital of Philadelphia, Philadelphia, PA; Mary Sokoloski, M.D., St. Christopher's Hospital for Children, Philadelphia, PA; Lee Beerman, M.D., Children's Hospital of Pittsburgh, Pittsburgh, PA; Marc LeGras, M.D., Emanuel Children's Hospital, Portland, OR; Seshadri Balaji, M.D., University of Oregon Health Sciences Center, Portland, OR; Co-burn Porter, M.D., Mayo Clinic, Rochester, MN; Jeanny Park, M.D., University of California Davis, Sacramento, CA; Susan Etheridge, M.D., University of Utah Primary Children's Hospital, Salt Lake City, UT; James C. Perry, M.D., Children's Hospital San Diego, San Diego, CA; George F. Van Hare, M.D., University of California San Francisco, San Francisco, CA; Frank Cecchin, M.D., Children's Hospital Seattle, Seattle, WA; Frank Zimmerman, M.D., St. Louis Children's Hospital, St. Louis, MO; Burt Bromberg, M.D., Children's Heart Center, St. Louis, St. Louis, MO; Craig Byrum, M.D., SUNYSyracuse, Syracuse, NY; Ricardo Samson, M.D., University of Arizona, Tucson, AZ; Robert Hamilton, M.D., Hospital for Sick Children, Toronto, Toronto, Ontario, Canada; Jeff Moak, M.D., Children's Hospital National Medical Center, Washington, DC.

\section{Appendix II}

\section{Diagnostic Criteria}

\section{Accessory Pathway Tachycardia}

Must meet A, B, or C:

A. Preexcitation, defined as (1) short PR interval, short HV interval, and long QRS, or (2) progressively shorter HV and longer QRS with incremental atrial pacing. [el6] 
B. Narrow QRS tachycardia, characterized by (1) eccentric retrograde atrial activation during supraventricular tachycardia (SVT) with shortest ventriculoatrial (VA) time $>60 \mathrm{~ms}$, or (2) noneccentric retrograde atrial activation during SVT with shortest VA time $>60 \mathrm{~ms}$, and nondecremental VA conduction during incremental ventricular pacing.[el6]

C. Persistent junctional reciprocating tachycardia, characterized by (1) incessant narrow QRS tachycardia, (2) earliest retrograde atrial activation in region of coronary sinus ostium, and (3) terminates with adenosine.

\section{Atrioventricular Nodal Reentrant Tachycardia}

Narrow QRS tachycardia, characterized by a short ventriculoatrial (VA) time $(<40 \mathrm{~ms}$ at earliest atrial site), and (1) termination with adenosine, or (2) dual atrioventricular (AV) nodal physiology (50-ms jump in $\mathrm{A}_{1} \mathrm{H}_{2}$ with a 10-ms decrement in $\mathrm{A}_{1} \mathrm{~A}_{2}$ ).

\section{Appendix III}

\section{Participating Investigators in Pilot Trial}

J. Philip Saul, M.D., Children's Hospital, Boston, MA; Richard Friedman, M.D., Texas Children's Hospital, Houston, TX; Craig Byrum, M.D., State University of New York-Syracuse Health Science Center, Syracuse, NY; Michael Schaffer, M.D., Denver Children's Hospital, Denver, CO; George F. Van Hare, M.D., Rainbow Babies and Children's Hospital, Cleveland, OH; John Kugler, M.D., University of Nebraska, Omaha, NE; Ann Dunnigan, M.D., (reviewer) Minneapolis Heart Institute, Minneapolis, MN. 